\title{
INFLUÊNCIA DA CALCINAÇÃO COMPLETA DO METACAULIM NA RESISTÊNCIA MECÂNICA DE ARGAMASSAS GEOPOLIMÉRICAS*
}

\author{
Thais da Silva Rocha ${ }^{1}$ \\ Dylmar Penteado Dias ${ }^{2}$ \\ Fernando César Coelho França ${ }^{3}$ \\ Sérgio Neves Monteiro ${ }^{4}$ \\ Afonso Rangel Garcez de Azevedo 5 \\ Rafael Rangel de Salles Guerra ${ }^{6}$
}

\section{Resumo}

O objetivo deste trabalho é avaliar a reatividade de um metacaulim comercial a partir da análise de sua cristalinidade. Um tratamento térmico é proposto a fim de tornar o material mais amorfo e para isso o metacaulim comercial já calcinado foi submetido a três tempos e temperaturas de queima em forno mufla $\left(700^{\circ} \mathrm{C} \mathrm{em} 1 \mathrm{~h}, 600^{\circ} \mathrm{C} \mathrm{em}\right.$ $2 \mathrm{~h}$ e $500^{\circ} \mathrm{C}$ em $1 \mathrm{~h}$ ). Após a queima o tratamento térmico que alcançou o melhor resultado foi 0 de $600^{\circ} \mathrm{C}$ em 2 horas, apresentando quase que somente picos cristalinos de quartzo nas análises de DRX. Para avaliar a influência do amorfismo na resistência mecânica de argamassas geopoliméricas foram moldados corpos de prova cilíndricos de $\varnothing 50 \times 100 \mathrm{~mm}$ com o metacaulim comercial como referência e com os metacaulins calcinados. Todas as argamassas foram feitas com a mesma dosagem e ensaiadas com 24 horas após a moldagem. O aumento de resistência à compressão chegou a $53 \%$ com o metacaulim queimado a $600^{\circ} \mathrm{C}$, mostrando que assim como acontece com argamassas de cimento Portland a calcinação completa é um fator crucial para o ganho de resistência mecânica em argamassas geopoliméricas.

Palavras-chave: Argamassas geopoliméricas; Metacaulim; Calcinação; Resistência à compressão.

\section{INFLUENCE OF METAKAOLIN COMPLETE CALCINATION IN THE MECHANICAL STRENGTH OF GEOPOLIMERIC MORTARS}

\section{Abstract}

The objective of this work is to evaluate the reactivity of a commercial metakaolin from the analysis of its crystallinity. A heat treatment is proposed in order to make the material more amorphous and for this the commercial calcined metakaolin was subjected to three times and temperatures of burning in a muffle furnace $\left(700^{\circ} \mathrm{C}\right.$ in $1 \mathrm{~h}, 600^{\circ} \mathrm{C}$ in $2 \mathrm{~h}$ and $500^{\circ} \mathrm{C}$ in $1 \mathrm{~h}$ ). After the heat treatment, which reached the best result, it was $600^{\circ} \mathrm{C}$ in 2 hours, presenting almost only crystalline quartz peaks in the XRD analyzes. In order to evaluate the influence of amorphism on the mechanical strength of geopolymeric mortars, cylindrical specimens of 50×100 mm were molded with the commercial metakaolin as reference and the calcined metakaolin. All mortars were made with the same dosage and tested 24 hours after molding. The increase in compressive strength reached $53 \%$ with metakaolin burned at $600^{\circ} \mathrm{C}$, showing that as with Portland cement mortars, complete calcination is a crucial factor for the gain of mechanical resistance in geopolymeric mortars.

Keywords: Geopolymeric mortars; Metakaolin; Calcination; Compressive strength.

1 Engenheira Civil, mestranda em Engenharia Civil, LECIV, Universidade Estadual do Norte Fluminense Darcy Ribeiro, Campos dos Goytacazes, Rio de Janeiro, Brasil. 
2 Engenheiro Civil, doutor em Ciências dos Materiais, professor associado, CCT/LECIV, Universidade Estadual do Norte Fluminense Darcy Ribeiro, Campos dos Goytacazes, Rio de Janeiro, Brasil.

3 Matemático, doutorando em Engenharia Civil, CCT/LECIV, Universidade Estadual do Norte Fluminense Darcy Ribeiro, Campos dos Goytacazes, Rio de Janeiro, Brasil .

4 Engenheiro Civil, doutorando em Engenharia Civil, CCT/LECIV, Universidade Estadual do Norte Fluminense Darcy Ribeiro, Campos dos Goytacazes, Rio de Janeiro, Brasil.

5 Engenheiro Metalúrgico, doutor em Ciências dos Materiais e Engenharia, professor titular, Instituto Militar de Engenharia, Rio de Janeiro, Rio de Janeiro, Brasil.

6 Engenheiro de Exploração e Produção de Petróleo, graduado, CCT/LENEP, Universidade Estadual do Norte Fluminense Darcy Ribeiro, Campos dos Goytacazes, Rio de Janeiro, Brasil. 


\section{INTRODUÇÃO}

O geopolímero é um composto formado por uma fonte de $\mathrm{SiO}_{2}$ e $\mathrm{Al}_{2} \mathrm{O}_{3}$ amorfos e uma solução alcalina ativadora em formulações pré-determinadas. As matérias-primas utilizadas em geopolímeros são basicamente fontes de aluminossilicatos e um ativador alcalino. Os aluminossilicatos devem apresentar uma quantidade considerável de material amorfo, assim como uma dimensão granulométrica adequada. Já o ativador deve apresentar $\mathrm{pH}$ elevado o suficiente para conseguir dissolver a parcela amorfa do precursor sólido [1].

Os aluminossilicatos mais utlizados são as escórias de alto-forno, as argilas calcinadas e as cinzas da queima do carvão mineral. A argila mais utilizada é o caulim que, a partir de ativação térmica, forma o metacaulim. O metacaulim é um material pozolânico amorfo (de estrutura cristalina desordenada), obtido por processo industrial de ativação térmica entre 600 e $850^{\circ} \mathrm{C}$ e moagem de argilas cauliníticas e caulins. Essas matérias-primas são compostas predominantemente por silicato de alumínio hidratado $\left[\mathrm{Al}_{2} \mathrm{Si}_{2} \mathrm{O}_{5}(\mathrm{OH})_{4}\right]$, que perdem os íons hidroxila de sua estrutura cristalina com a calcinação, transformando-se em metacaulinita $\left(\mathrm{Al}_{2} \mathrm{Si}_{2} \mathrm{O}_{7}\right)$, composto constituído essencialmente por partículas lamelares com estrutura predominantemente não cristalina e com elevada finura [2].

A desidroxilação da caulinita ocorre conforme a Equação 1:

$$
\mathrm{Al}_{2} \mathrm{O}_{3} .2 \mathrm{SiO}_{2} .2 \mathrm{H}_{2} \mathrm{O} \rightarrow \mathrm{Al}_{2} \mathrm{O}_{3} .2 \mathrm{SiO}_{2}+2 \mathrm{H}_{2} \mathrm{O}
$$

Esta transformação ocorre pela perda de água da caulinita, dando origem a uma estrutura amorfa. Se o processo de calcinação continua, a elevação da temperatura provoca uma recristalização da fase amorfa da metacaulinita para uma fase cristalina intermediária, chamada de espinélio, até a nucleação da mulita [3].

Segundo Davidovits [4], com a transformação do caulim em metacaulim o material se torna muito mais reativo, com propriedades pozolânicas. Além disso, o produto obtido pode ter diferentes graus de finura, superfície específica e também apresentar colorações variadas.

O metacaulim foi muito estudado devido a sua atividade pozolânica e efeito microfiler, sendo amplamente utilizado na produção de concretos de alto desempenho. Quando aplicado na produção de geopolímeros, o material resultante é menos impuro e de mais fácil caracterização, sendo considerado um "sistema modelo".

Entretanto, muitos fatores influenciam na reatividade do metacaulim e consequentemente na sua qualidade para essas aplicações, dentre eles: a granulometria do material; o tipo de forno, o tempo de permanência, as taxas de aquecimento e o resfrimento após calcinação.

Com o intuito de produzir argamassas geopoliméricas de elevada resistência mecânica e tendo a qualidade do metacaulim como um fator primordial para isso, 0 objetivo deste trabalho é estudar um metacaulim comercial e avaliar se a sua calcinação foi suficiente para produzir um material amorfo, logo reativo. Após a análise do metacaulim, um tratamento térmico é proposto para alcançar as propriedades descritas e a resistência das argamassas com os metacaulins calcinados é avaliada. 


\section{MATERIAIS E MÉTODOS}

O metacaulim desta pesquisa é um produto comercial produzido pela empresa Metacaulim do Brasil (Jundiaí - S.P.). Sua composição química foi determinada a partir da análise semi-quantitativa por espectrometria de fluorescência de raios-X e está apresentada juntamente com as características físicas na Tabela 1.

Tabela 1. Características físicas e químicas do metacaulim

\begin{tabular}{|c|c|}
\hline $\begin{array}{c}\text { Características físicas e } \\
\text { químicas }\end{array}$ & Metacaulim (MC) \\
\hline $\mathrm{SiO}_{2}(\%)$ & 48,43 \\
\hline $\mathrm{Al}_{2} \mathrm{O}_{3}(\%)$ & 44,76 \\
\hline $\mathrm{Fe}_{2} \mathrm{O}_{3}(\%)$ & 2,39 \\
\hline $\mathrm{CaO}(\%)$ & 0,10 \\
\hline $\mathrm{SO}_{3}(\%)$ & 1,24 \\
\hline $\mathrm{K}_{2} \mathrm{O}(\%)$ & 1,51 \\
\hline $\mathrm{TiO}_{2}(\%)$ & 1,45 \\
\hline Perda ao Fogo (\%) ${ }^{a}$ & 3,33 \\
\hline Massa específica $\left(\mathrm{g} / \mathrm{cm}^{3}\right)^{b}$ & 2,65 \\
\hline
\end{tabular}

O ensaio de granulometria a laser foi realizado a fim de se obter a curva de distribuição granulométrica do metacaulim. Para a realização do ensaio foi utilizado o equipamento CILAS1190 do Laboratório de Geotecnia e Meio Ambiente da PUCRio. As amostras foram dispersas em água destilada e as condições de ensaio foram: agitação de $1500 \mathrm{rpm}$, tempo de ultrasom de $5 \mathrm{~min}$, obscuração por volta de $20 \%$ e tempo de dispersão de 5 minutos. A Figura 1 apresenta a curva granulométrica do metacaulim. Os diâmetros das partículas do metacaulim são definidos como os tamanhos de partículas abaixo dos quais se encontram $10 \%$ $\left(D_{10}\right), 50 \%\left(D_{50}\right)$ e $90 \%\left(D_{90}\right)$ de massa do material. Esses diâmetros se encontram na Tabela 2.

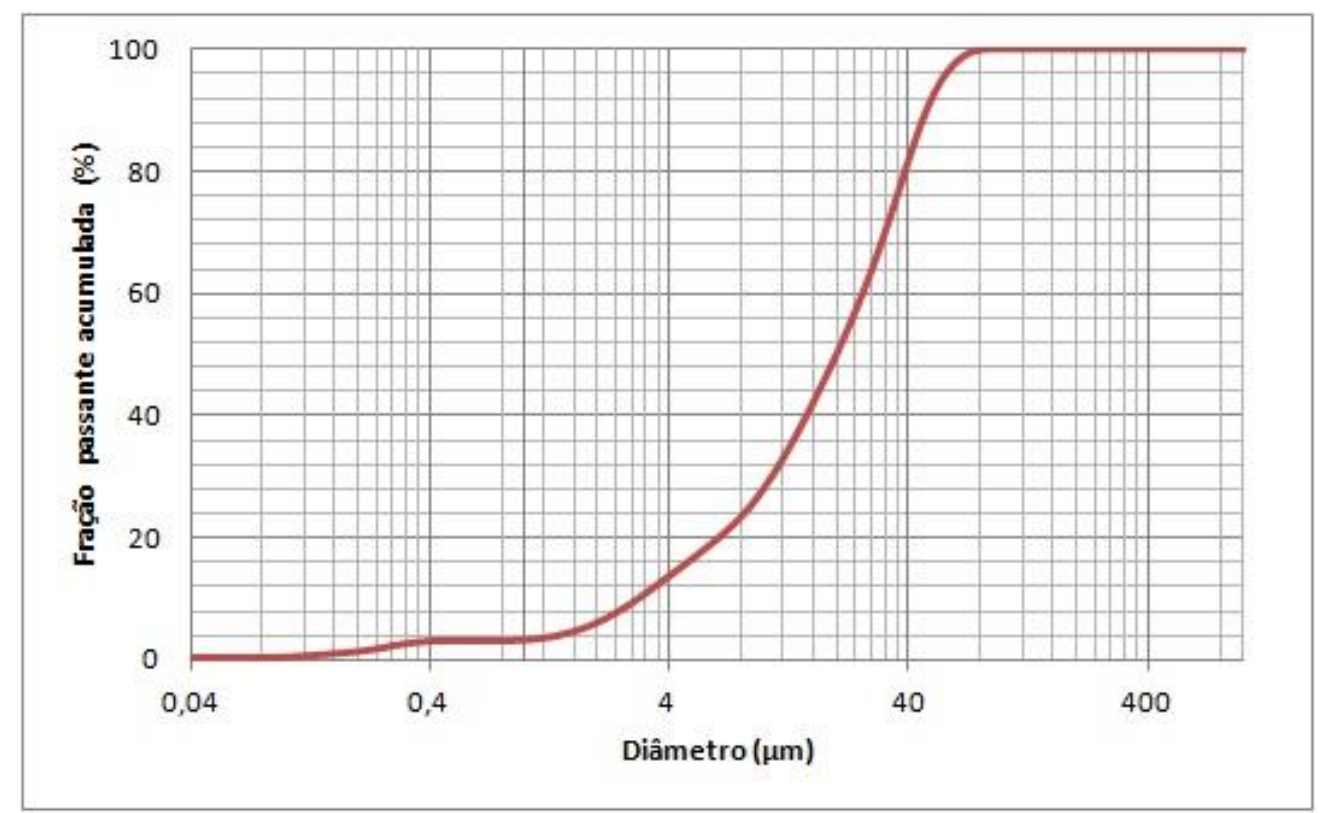

Figura 1. Curva granulométrica do metacaulim. 
Tabela 2. Diâmetros das partículas do metacaulim

\begin{tabular}{cc}
\hline Diâmetro & Metacaulim (MC) \\
\hline$D_{10}$ & $2,93 \mu \mathrm{m}$ \\
\hline$D_{50}$ & $18,82 \mu \mathrm{m}$ \\
\hline$D_{90}$ & $47,34 \mu \mathrm{m}$ \\
\hline$D_{\text {médio }}$ & $23,04 \mu \mathrm{m}$ \\
\hline
\end{tabular}

A identificação das fases cristalinas do metacaulim foi realizada no Laboratório de Engenharia de Materiais Avançados da Uenf (LAMAV) por meio de uma difatrometria de raios- $X$ no difratômetro de raios- $X$ da marca Shimadzu, XRD7000 , com radiação de cobre (Cu-ka, $\lambda=1,5418 \AA$ ), operando a $40 \mathrm{kV}$ e $30 \mathrm{~mA}$. As varreduras foram realizadas com velocidade angular de $0,02^{\circ}$ por segundo $e$ intervalo de medida entre os ângulos de Bragg $(2 \theta)$ de $5^{\circ}$ e $80^{\circ}$.

Visando remover os grupos estruturais de hidroxila (ou seja, fazer com que a caulinita se transforme em metacaulinita) de modo que ficasse somente identificável o quartzo, foi realizado um tratamento térmico nas amostras de metacaulim. Esse tratamento térmico consistiu em calcinar em um forno mufla $530 \mathrm{~g}$ de metacaulim nas temperaturas de $700^{\circ} \mathrm{C}$ por 1 hora; $600^{\circ} \mathrm{C}$ por 2 horas e $500^{\circ} \mathrm{C}$ por 3 horas. Ao término deste intervalo, as amostras foram deixadas dentro do forno resfriando até alcançar a temperatura ambiente. $\mathrm{O}$ equipamento empregado foi um forno mufla $\mathrm{FL}$ 1300 da marca Fornos Inti, com controlador de temperatura e precisão de $+/-5^{\circ} \mathrm{C}$, podendo alcançar temperaturas de até $1320^{\circ} \mathrm{C}$.

Após o resfriamento completo do metacaulim, foram moldados corpos de prova cilíndricos de $\varnothing 50 \mathrm{~mm} \times 100 \mathrm{~mm}$ de argamassas geopoliméricas com o metacaulim comercial e com os metacaulins calcinados. Todos as moldagens utilizaram a mesma dosagem objetivando avaliar se o aumento da reatividade do metacaulim influenciava na resistência à compressão dos geopolímeros como acontece com o cimento Portland.

Devido ao ganho elevado de resistência dos geopolímeros nas primeiras idades, optou-se por ensaiar todos os corpos de prova com 24h após a moldagem. O ensaio de resistência à compressão foi realizado de acordo com NBR 5730:2007 [7].

\section{RESULTADOS E DISCUSSÃO}

A Figura 2 apresenta os resultados da difração de raios-X para o metacaulim utilizado. O gráfico apresenta picos característicos de quartzo $\left(\mathrm{SiO}_{2}\right)$ e caulinita (ou silicato de alumínio hidratado $\left[\mathrm{Al}_{2} \mathrm{Si}_{2} \mathrm{O}_{5}(\mathrm{OH})_{4}\right]$ ), o que indica a presença de impurezas e calcinação incompleta.

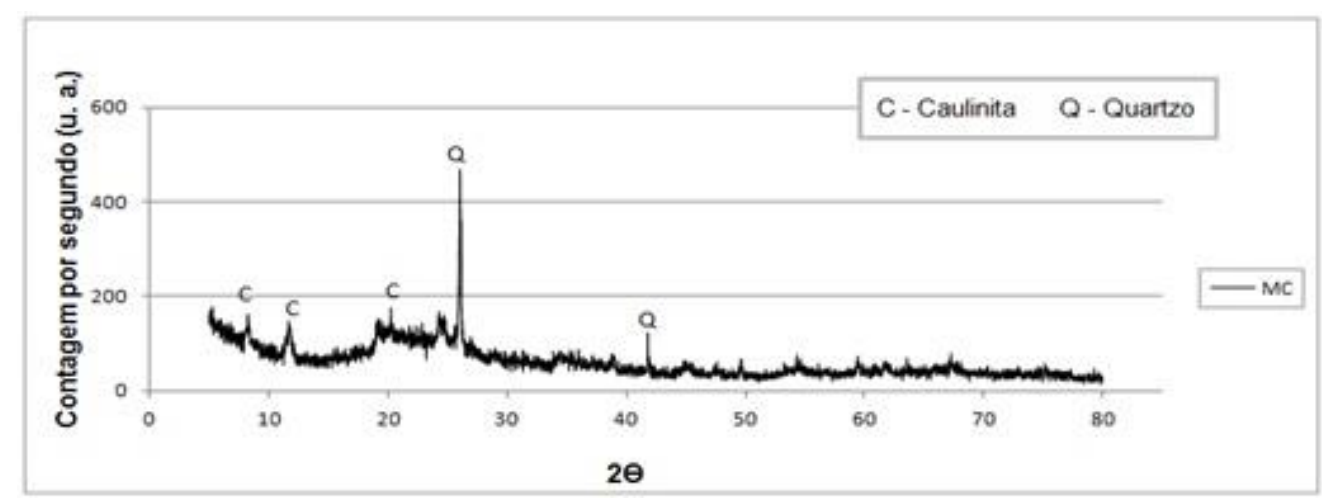

Figura 2. Difratograma do metacaulim. 
Após o tratamento térmico foram realizadas análises de DRX nas amostras calcinadas como mostra a Figura 3 . É possível perceber que o tratamento térmico mais eficaz foi o de $600^{\circ} \mathrm{C}$ por 2 horas. Nesta temperatura, quase todos os picos característicos de caulinita (ou silicato de alumínio hidratado $\left[\mathrm{Al}_{2} \mathrm{Si}_{2} \mathrm{O}_{5}(\mathrm{OH})_{4}\right]$ ) desapareceram devido a desordem estrutural causada pela desidroxilação das folhas octaédricas do aluminossilicato, permancendo identificável somente o quartzo e um pico de caulinita. Quanto maior o amorfismo do metacaulim mais reativo ele será, logo a menor incidência de picos característicos no gráfico de DRX, maior será o grau de vitrificação do material (menor cristalinidade).

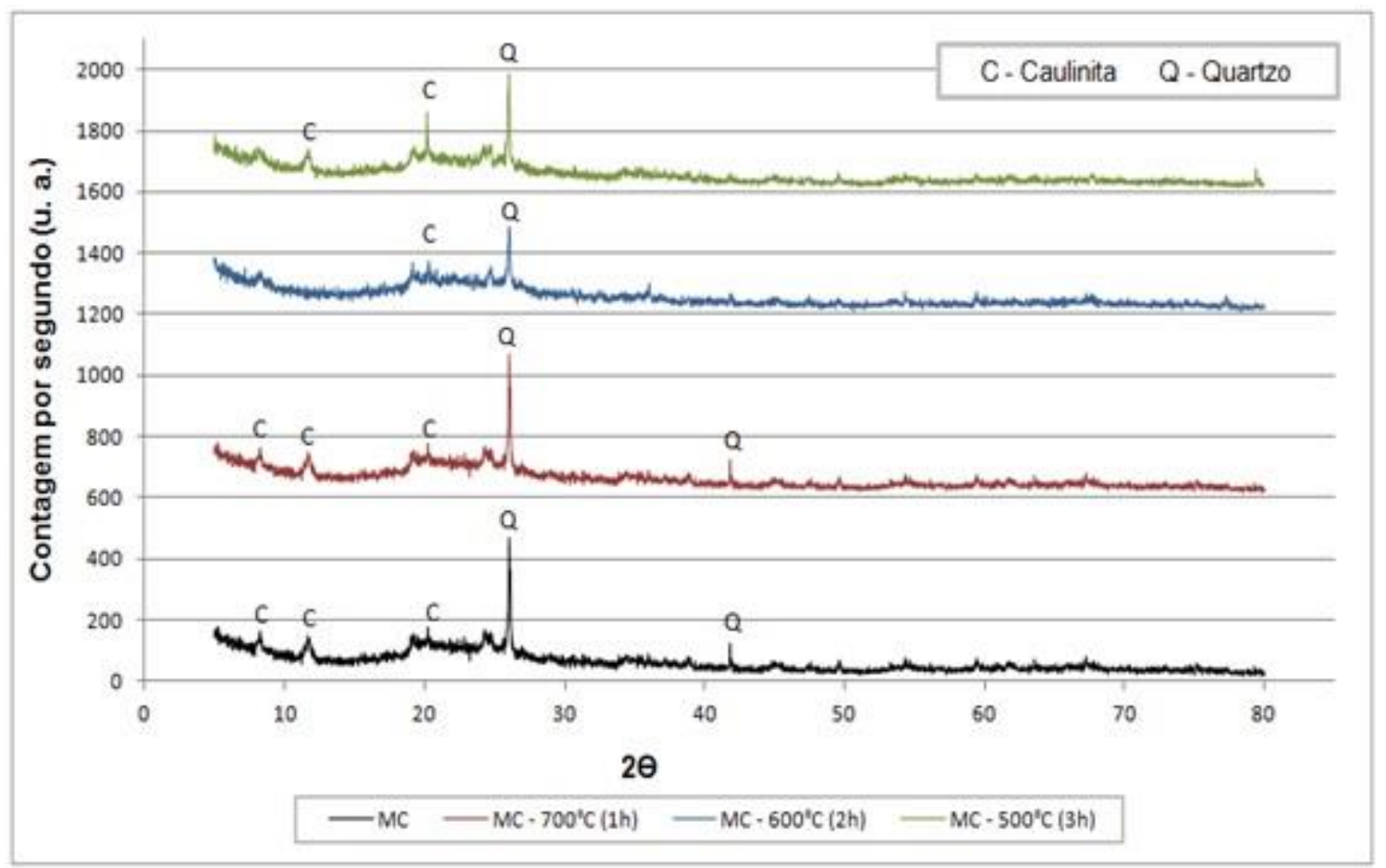

Figura 3. Difratograma do metacaulim antes e após tratamento térmico nas temperaturas de $700^{\circ} \mathrm{C}$ por 1 hora, $600^{\circ} \mathrm{C}$ por 2 horas e $500^{\circ} \mathrm{C}$ por 3 horas.

O tratamento térmico do metacaulim também causou no material uma mudança de coloração como pode ser observado na Figura 4. Neste caso o tempo de forno foi o fator que mais influenciou na mudança de cor, como pode ser observado a amostra que ficou 3 horas no forno foi a que ficou mais avermelhada.

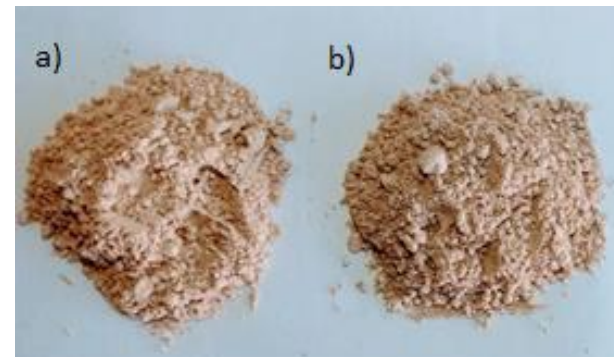

c)

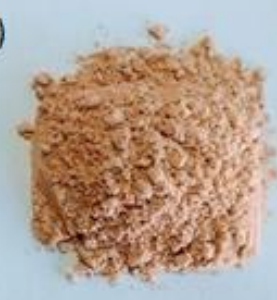

d)

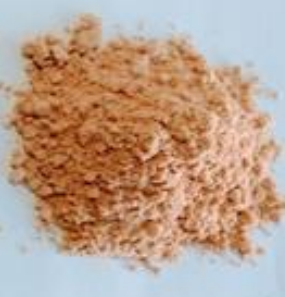

Figura 4. Metacaulim: a) antes do tratamento térmico; após tratamento térmico nas temperaturas de b) $700^{\circ} \mathrm{C}$ por 1 hora, c) $600^{\circ} \mathrm{C}$ por 2 horas e d) $500^{\circ} \mathrm{C}$ por 3 horas. 
A matriz geopolimérica foi produzida utilizando metacaulim como precursor e areia passante na peneira $1,18 \mathrm{~mm}$ como agregado miúdo e a relação de massa entre o precursor e o agregado foi de 1:1. As razões molares que foram atendidas para a dosagem da argamassa geopolimérica são: $\frac{\mathrm{SiO}_{2}}{\mathrm{Al}_{2} \mathrm{O}_{3}}=2,5 ; \frac{\mathrm{H}_{2} \mathrm{O}}{\mathrm{M}_{2} \mathrm{O}}=9,6 ; \frac{\mathrm{M}_{2} \mathrm{O}}{\mathrm{SiO}_{2}}=0,29$; $\frac{M_{2} \mathrm{O}}{\mathrm{Al}_{2} \mathrm{O}_{3}}=\mathbf{0 , 8 6}$. A Figura 5 apresenta os resultados de resistência à compressão axial em 24 horas após a moldagem para a argamassa moldada com o metacaulim comercial (MC) e as demais com os metacaulins que sofreram tratamento térmico.

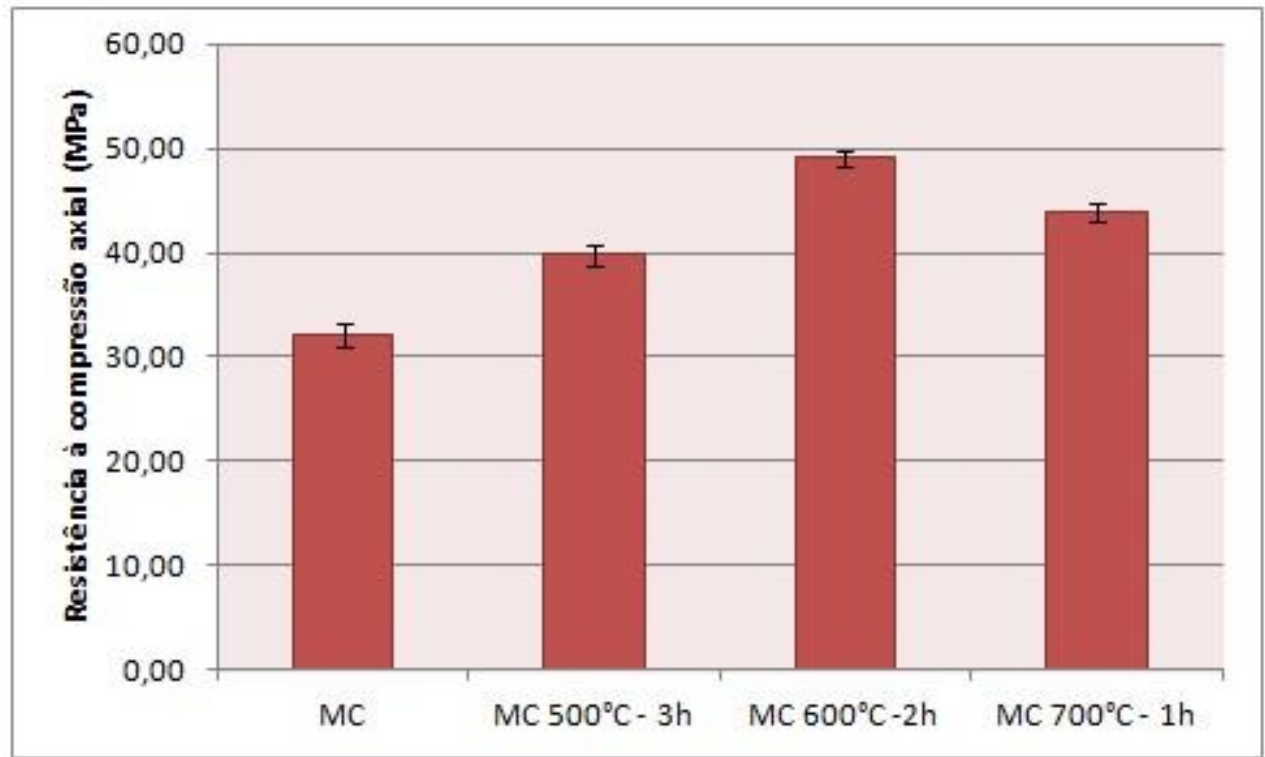

Figura 5. Resistência à compressão axial em 24 horas das argamassas com metacaulim comercial e calcinados.

É possível perceber que quanto maior foi o nível de amorfismo (ou vitrificação) do material maior foi sua reatividade, assim como na reatividade com o cimento Portland [8]. Para uma calcinação quase completa, como no caso do tratamento térmico a $600^{\circ} \mathrm{C}$ por 2 horas, o aumento de resistência à compressão para uma mesma dosagem no mesmo período de tempo foi de $53 \%$.

O processamento do metacaulim é de alto custo e este deve ser o motivo pelo qual a calcinação que foi feita em fábrica não tenha sido completa apesar do material se enquadrar nos requisitos de um metacaulim de alta reatividade.

O objetivo do trabalho foi o de avaliar somente a influência da calcinação completa do metacaulim na resistência à compressão de geopolímeros. Entretanto, o ideal seria após a calcinação realizar uma nova moagem, pois durante o tratamento térmico ocorre aglomeração de partículas que resulta em um aumento do seu diâmetro médio. Além disso, uma maior superfície específica do material promove um aumento na velocidade de reação durante a geopolimerização, e consequentemente uma resistência mecânica inicial mais elevada.

\section{CONCLUSÃO}

O tratamento que alcançou o maior nível de amorfismo de acordo com análises de $\mathrm{DRX}$ foi o de $600^{\circ} \mathrm{C}$ por 2 horas. Corroborando com as análises de $\mathrm{DRX}$, as resistências à compressão das argamassas geopoliméricas moldadas com os metacaulins calcinados foram em todos os casos maiores que as moldadas com 0 metacaulim comercial, em especial para o melhor tratamento térmico, alcançando 
$53 \%$ a mais de resistência. Apesar do alto custo de processamento do metacaulim, caso seja considerado viável, o ganho que resistência causado é muito representativo.

\section{Agradecimentos}

Agradeço à Fundação Carlos Chagas de Amparo à Pesquisa do Estado do Rio de Janeiro (Faperj) pela bolsa de pesquisa concedida durante o período de 2015 a 2017, e aos Laboratórios da PUC-Rio e LAMAV (UENF) pela realização dos ensaios.

\section{REFERÊNCIAS}

1 Longhi, Márlon Augusto. Álcali-ativação do lodo de caulim calcinado e cinza pesada com ativadores convencionais e silicato de sódio alternativo. Dissertação de Mestrado. Rio grande do Sul: Universidade Federal do Rio Grande do Sul; 2005.

2 Cunha, M. F.; Forti, B. A.; Silva B. I. Tendências da normalização de metacaulim para utilização em concreto de alto desempenho. Reunião Anual do Ibrancon 52 - Novas Tecnologias do Concreto para o Crescimento Sustentável, 2010; Anais IBRACON. 2010: 46-53.

3 Mothé, L. P.. Avaliação das condições ideais para a produção de metacaulinita através da análise da sua atividade pozolânica. Dissertação de Mestrado. Campos dos Goytacazes: Universidade Estadual do Norte Fluminense Darcy Ribeiro; 2004.

4 Davidovits, J.; Properties of geopolymer cements, Alkaline Cements and Concretes, Kiev, Ukraine. 1994: 131-149.

5 Associação Mercosul de Normalização. NM 18: Cimento Portland - Análise Química - Perda ao Fogo. AMN, 2012.

6 Associação Mercosul de Normalização. NM 23: Cimento Portland e outros materiais em pó - Determinação da Massa Específica. AMN, 2001.

7 Associação Brasileira de Normas Técnicas. NBR 5739: Concreto - Ensaio de compressão de corpos-de-prova cilíndricos - Método de ensaio. ABNT, 1994.

8 Rocha, G. G. N. Caracterização Microestrutural do Metaculim de Alta Reatividade. Dissertação de Mestrado. Belo Horizonte: Universidade Federal de Minas Gerais; 2005. 